\title{
Job Satisfaction and Its Associated Factors Among General Practitioners in China
}

\author{
Liqing Li, PhD, Yong Gan, PhD, Heng Jiang, PhD, Yudi Yang, MS, Xiaogang \\ Zhou, PhD, Yanling Zheng, MS, Fang Yu, BS, Jianxin Liu, MS, Yanyan Zhong, MS, \\ Yanli Chen, BS, Minyi Yu, MS, Ling Liu, BS, Junan Liu, PbD, and Zuxun Lu, PhD
}

Objectives: Investigation is scare on job satisfaction among general practitioners (GPs) in China. This study aimed to investigate job satisfaction of GPs in China and explore its determinants.

Methods: A multistage-stratified random sampling method was used to collect data with a structured self-administered questionnaire from 3236 GPs (response rate, 99.8\%) working in community health institutions in China between October 2017 and February 2018. Multiple linear stepwise regression analysis was used to analyze the associated factors with job satisfaction among GPs.

Results: Among these respondents, 1215 (37.5\%), 352 (10.9\%), and 1669 (51.6\%) GPs were dissatisfied, moderate, and satisfied for their current job, respectively. Male GPs, a higher education level, at a higher professional title, at a lower level of income, and those with heavy work stress had a lower job satisfaction. In addition, GPs who often worked overtime, who were at a higher level of emotional exhaustion, at a higher level of depersonalization, at a lower level of personal accomplishment, and who had less occupational development opportunities reported a lower level of job satisfaction.

Conclusion: These findings suggest that job satisfaction among Chinese GPs is at a moderate level. Region, sex, professional title, education level, working overtime, income level, emotional exhaustion, depersonalization, personal accomplishment, work stress, and occupational development opportunities were significant predictors of job satisfaction. (J Am Board Fam Med 2020;33:456-459.)

Keywords: China, Depersonalization, General Practitioners, Job Satisfaction, Occupational Stress, Public Health, Regression Analysis, Surveys and Questionnaires

General practitioner (GP) shortages and high turnover have become concerns in developed and

This article was externally peer reviewed.

Submitted 4 April 2019; revised 6 July 2019; accepted 9 July 2019.

From the Department of Management Science and Engineering, School of Economics and Management, Jiangxi Science and Technology Normal University, Nanchang, Jiangxi, China (L. Li); Department of Social Medicine and Health Management, School of Public Health, Tongji Medical College, Huazhong University of Science and Technology, Wuhan, Hubei, China (L. Li, YG, YY, Jianxin Liu, Junan Liu, ZL); Centre for Alcohol Policy Research, School of Psychology and Public Health, La Trobe University, Melbourne, Victoria, Australia (HJ); Melbourne School of Population and Global Health, University of Melbourne, Melbourne, Victoria, Australia (HJ); School of Economics and Management, East China JiaoTong University, Nanchang, Jiangxi, China (XZ); Shouyilu Street Community Health Service Center of Wuchang District, Wuhan, Hubei, China (Y. Zheng, FY, YC, MY, L. Liu); Huazhong University of Science and Technology Hospital, Wuhan, Hubei, China (Y. Zhong).

Authors' contributions: L. Li, YG, and ZL conceived and designed the study. L. Li, YG, YY, XZ, Y. Zheng, FY, YC, Jianxin Liu, Y. Zhong, MY, and L. Liu participated in the developing countries. ${ }^{1,2}$ Previous research indicated that job dissatisfaction was associated with turnover

acquisition of data. L. Li, YG, and YY analyzed the data. L. $\mathrm{Li}$ and YG drafted the manuscript, and L. Li L, YG, HJ, YY, and $\mathrm{ZL}$ revised the manuscript. All authors read and approved the final manuscript. Junan Liu and ZL are the guarantors of this work and had full access to all the data in the study and take responsibility for its integrity and the accuracy of the data analysis.

Authors Liqing Li and Yong Gan contributed equally to this work.

Funding: This study was supported by the National Social Science Foundation of China (grant no. 18ZDA085). The funders had no role in study design, data collection and analysis, decision to publish, or preparation of the manuscript.

Competing interests: We declared that we have no conflict of interests.

Ethics statement: The study protocol was approved by the institutional review boards of Tongji Medical College, Huazhong University of Science and Technology, Wuhan, China.

Corresponding authors: Professor Zuxun Lu or Professor Junan Liu, Department of Social Medicine and Health Management, School of Public Health, Tongji Medical College, Huazhong University of Science and Technology, No. 13 Hangkong Road, Wuhan 430030, China (E-mail: zuxunlu@ yahoo.com orlja10_11@163.com). 
intentions among GPs. ${ }^{3,4}$ Job satisfaction was important for both GPs and the quality of the service they delivered. ${ }^{5}$ Studies on investigating job satisfaction of health care workers (including GPs) and its determinants have been conducted in many developed countries. ${ }^{2,6,7}$ However, no study has analyzed the job satisfaction among GPs and its predictors based on a national representative sample in China. Therefore, this study aimed to investigate the level of job satisfaction and associated factors among Chinese GPs.

A cross-sectional study was conducted between 10 October 2017 and 28 February 2018 in China. A multistage-stratified random sampling survey was used in the study. First, 4 provinces each from eastern (Shanghai, Beijing, Guangdong, and Zhejiang), central (Hubei, Anhui, Heilongjiang, and Heinan), and west China (Sichuan, Chongqing, Guizhou, and Yunan) were randomly selected. Second, 30 community health institutions (CHIs) were randomly selected from each province. Finally, according to the number of GPs and scales of CHIs, from each sampled CHI, $40 \%$ of the on-post GPs with at least 1 year of work experience were randomly selected to complete a self-administered questionnaire. In total, 3244 GPs participated in the survey,

Table 1. Multiple Linear Stepwise Regression Analysis for Association with Job Satisfaction among GPs (n = 3236)*

\begin{tabular}{|c|c|c|c|c|}
\hline Variables & $\beta$ & $\mathrm{SE}$ & $t$ & $P$ Value \\
\hline \multicolumn{5}{|l|}{ Region (ref = western China) } \\
\hline Eastern China & 0.599 & 0.201 & -2.968 & .003 \\
\hline \multicolumn{5}{|l|}{ Sex $(r e f=$ female $)$} \\
\hline Male & -0.537 & 0.197 & -2.720 & .007 \\
\hline \multicolumn{5}{|c|}{ Education level $\left(\mathrm{ref}=\right.$ associate's degree or vocational diploma $\left.{ }^{\dagger}\right)$} \\
\hline Bachelor degree & -0.975 & 0.217 & -4.491 & $<.0001$ \\
\hline Master degree or higher & -1.633 & 0.444 & -3.678 & $<.0001$ \\
\hline \multicolumn{5}{|l|}{ Working overtime (ref = never) } \\
\hline Frequent & -1.135 & 0.203 & -5.606 & $<.0001$ \\
\hline \multicolumn{5}{|l|}{ Income level (ref = moderate or below) } \\
\hline Moderate & 2.374 & 0.218 & 10.866 & $<.0001$ \\
\hline Moderate or higher & 2.660 & 0.576 & 4.622 & $<.0001$ \\
\hline \multicolumn{5}{|l|}{ Professional title (ref = elementary or below) } \\
\hline Intermediate & -0.803 & 0.208 & -3.863 & $<.0001$ \\
\hline Senior & -0.945 & 0.319 & -2.968 & .003 \\
\hline Emotional exhaustion (1 score increase) & -0.110 & 0.009 & -12.003 & $<.0001$ \\
\hline Depersonalization (1 score increase) & -0.074 & 0.018 & -4.137 & $<.0001$ \\
\hline Personal accomplishment (1 score increase) & 0.065 & 0.010 & 6.823 & $<.0001$ \\
\hline \multicolumn{5}{|l|}{ Work stress $($ ref $=$ low $)$} \\
\hline Intermediate & -1.089 & 0.342 & -3.187 & .001 \\
\hline High & -1.738 & 0.344 & -5.059 & $<.0001$ \\
\hline \multicolumn{5}{|c|}{ Occupational development opportunities (ref $=$ fewer $)$} \\
\hline General & 3.821 & 0.197 & 19.429 & $<.0001$ \\
\hline More & 7.721 & 0.372 & 20.742 & $<.0001$ \\
\hline Intercept & 35.143 & 0.495 & 70.992 & $<0.0001$ \\
\hline
\end{tabular}

*Adjustment for marital status (unmarried, widowed, divorced, or married), manager responsibility (yes, no), contract status (permanent, temporary), age (continuous), work tenure (continuous), and other variables in the models. $R^{2}=0.365 ; F=117.094, P<.0001$. ${ }^{\dagger}$ GPs who have acquired an associate's degree or vocational diploma. An associate's degree required 3 years of education in college after graduation from senior middle school (grade year 10 to year 12) or 5 years of education in college after graduation from junior middle school (grade year 7 to year 9). A vocational diploma requires 2 years of education in vocational school after graduation from senior middle school or 3 years of education in vocational school after graduation from junior middle school. The $\beta$ across each variable (including region, sex, education level, working overtime, income level, professional title, emotional exhaustion, depersonalization, personal accomplishment, work stress, and occupational development opportunities) was compared to generate the statistics. GP, general practitioner; ref, reference. 
of whom 8 GPs did not respond. Ultimately, 3236 eligible questionnaires were included in this analysis, yielding a response rate of $99.75 \%$.

We measured the job satisfaction developed by Shi et $\mathrm{al}^{8}$ consisting 11 items with a 5 -point Likert scale. Predictors included age, region, sex, marital status, education level, contract status, manager responsibility, working overtime, income level, professional title, emotional exhaustion, depersonalization, personal accomplishment, work stress, and occupational development opportunities. Multiple linear stepwise regression analysis was used to estimate the associated factors for job satisfaction among GPs (level for selection and elimination: $P=.05$ and $P=.10$, respectively). Analyses were performed using SAS 9.2, and all tests were 2 sided with a significance level of 0.05 .

A total of 3236 GPs were included in our study (63.8\% females). The mean age of respondents was 37.42 years $(\mathrm{SD}=7.92)$. A total of $1229(38.0 \%)$ GPs were from eastern China, $67.5 \%$ of GPs had a permanent work contract, and $70.5 \%$ were at in the middle and lower income level groups.

The results of the multiple linear stepwise regression analysis show that those GPs who were working in eastern China; were female; had a junior college or below degree; were not working overtime; had higher income; were elementary or below; had a lower professional title; had low emotional exhaustion, low depersonalization, and high personal accomplishment; had fewer work pressures; and had more occupational development opportunities reported a higher level of job satisfaction (Table 1).

Over half of GPs $(51.6 \%)$ had higher levels of job satisfaction. The figure of high job satisfaction in this study is in the middle of reporting rates found in developed countries (eg, Germany, ${ }^{2}$ Canada, ${ }^{9}$ and the United Kingdom ${ }^{10}$ ) ranging from $42 \%$ to $72 \%$. The differences might be partly attributable to the participants' characteristics (ie, age, income, practice setting, rural/urban areas, sample size, and the measurement of job satisfaction).

Male GPs had a lower level of job satisfaction than female GPs, which is in line with some previous studies. ${ }^{11,12}$ Different job expectations and values in job rewards may cause the differences in job satisfaction between males and females. In addition, previous studies indicated that males and females have different values concerning the key factors in their jobs. Males emphasize promotion prospects; in contrast, females were more concerned with the good relations with colleagues and managers, the work content, and the working hours. ${ }^{13}$

With fewer work pressures, a higher job satisfaction was found among Chinese GPs. Our results were also compatible with previous studies. ${ }^{14}$ Work stress was a powerful driving force and was a negative factor affecting job performance and occupational activity. Therefore, reducing work stress is an effective way to improve the job satisfaction among GPs.

GPs with fewer occupational development opportunities had a lower job satisfaction. Fewer occupational development opportunities may drive GPs away from primary care, and more occupational development opportunities could help to attract more GPs. Previous studies supported our result that fewer occupational development opportunities may reduce efficiency, cut enthusiasm, or even lead to staff turnover. ${ }^{15}$

The Global Conference on Primary Health Care and the Health China 2030 of the Chinese government has stated that it is important to strengthen the construction of the primary health care infrastructure and delivery ability. Actions such as reducing the work intensity, increasing the wages of GPs, and providing career development opportunities for GPs are warranted to improve the level of job satisfaction of GPs.

The authors thank the GPs who participated in this research and staff members of Community Health Association of China involved in this study for their efforts in data collection.

To see this article online, please go to: http://jabfm.org/content/ 33/3/456.full.

\section{References}

1. Sibbald B, Bojke C, Gravelle H. National survey of job satisfaction and retirement intentions among general practitioners in England. $\mathrm{Br} \mathrm{Med} \mathrm{J}$ 2003;326:22-4.

2. Leutgeb R, Frankenhauser-Mannuss J, Scheuer M, Szecsenyi J, Goetz K. Job satisfaction and stressors for working in out-of-hours care-a pilot study with general practitioners in a rural area of Germany. BMC Fam Pract 2018;19:95.

3. Mein G, Martikainen P, Stansfeld SA, Brunner EJ, Fuhrer R, Marmot MG. Predictors of early retirement in British civil servants. Age Ageing 2000; 29:529-36.

4. Sibbald B, Enzer I, Cooper C, Rout U, Sutherland V. GP job satisfaction in 1987, 1990 and 1998: lessons for the future? Fam Pract 2000;17:364-71. 
5. Zhang X, Fang P. Job satisfaction of village doctors during the new healthcare reforms in China. Aust Health Review 2016;40:225-33.

6. Noroxe KB, Pedersen AF, Bro F, Vedsted P. Mental well-being and job satisfaction among general practitioners: a nationwide cross-sectional survey in Denmark. BMC Fam Pract 2018;19:130

7. Roos M, Watson J, Wensing M, Peters-Klimm F. Motivation for career choice and job satisfaction of GP trainees and newly qualified GPs across Europe: a seven countries cross-sectional survey. Educ Prim Care 2014;25:202-10.

8. Shi L, Song K, Rane S, Sun X, Li H, Meng Q. Factors associated with job satisfaction by Chinese primary care providers. Prim Health Care Res Dev 2014;15:46-57.

9. Malhotra J, Wong E, Thind A. Canadian family physician job satisfaction is it changing in an evolving practice environment? An analysis of the 2013 National Physician Survey database. BMC Fam Pract 2018;19:100.
10. Whalley D, Bojke C, Gravelle H, Sibbald B. GP job satisfaction in view of contract reform: a national survey. Br J Gen Pract 2006;56:87-92.

11. Sloane PJ, Williams H. Job satisfaction, comparison earnings, and gender. Labour 2000;14:473-502.

12. Carrillo-Garcia C, Solano-Ruiz MD, MartinezRoche ME, Gomez-Garcia CI. Job satisfaction among health care workers: the role of gender and age. Rev Lat-Am Enferm 2013;21:1314-20.

13. Zou M. Gender, work orientations and job satisfaction. Work Employ Soc 2015;29:3-22.

14. Kuusio H, Heponiemi T, Vanska J, Aalto AM, Ruskoaho J, Elovainio M. Psychosocial stress factors and intention to leave job: differences between foreign-born and Finnish-born general practitioners. Scand J Public Health 2013;41:405-11.

15. Lu Y, Hu XM, Huang XL, et al. The relationship between job satisfaction, work stress, work-family conflict, and turnover intention among physicians in Guangdong, China: a cross-sectional study. BMJ Open 2017;7:e014894. 